\title{
Future PHENIX Calorimetry and Trigger Upgrades
}

\author{
Anselm Vossen* \\ University of Illinois at Urbana-Champaign \\ E-mail: vossen@illinois.edu
}

In the near future, the PHENIX experiment at Brookhaven National Laboratory will install several upgrades to enable an extended physics program in the next decade. Centerpieces of this effort will be new vertex detectors, forward calorimetry and a muon trigger upgrade. This work will focus on the latter two. The planned forward calorimeter, FoCal, will bridge the gap in the acceptance between the currently installed electromagnetic calorimeters in PHENIX in the pseudorapidity range 1 to 3 . Using silicon tungsten technology, it will enable the measurement of direct photons and neutral mesons as well as jets in a kinematic domain that will allow access to low $x$ values in heavy ion and polarized proton collisions. This is especially true in $\sqrt{s}=500 \mathrm{GeV}$ proton-proton collisions. At these energies, a milestone in the RHIC spin program is the measurement of the sea quark polarization of protons via $\mathrm{W}$ production. Using the parity violating nature of the weak interaction, single spin asymmetries can be used to access this quantity at high momentum transfers and independent of quark fragmentation models. In order to detect muons from W decays, an upgrade of the PHENIX muon trigger is currently underway. It consists of new detector stations using RPC technology and new FEE for the existing muon tracker stations. 


\section{Introduction}

The PHENIX experiment [1] is located at the Relativistic Heavy Ion Collider (RHIC) at Brookhaven National Laboratory. RHIC has the capabilities to collide polarized protons and a wide variety of ions at center of mass energies up to $500 \mathrm{GeV}$ for protons and up to $200 \mathrm{GeV}$ per nucleon for other ions. After a very successful physics program over the last 10 years, PHENIX is currently planning and implementing several upgrades. Two of the upgrades, described in these proceedings, aim to measure the properties of the sea-quarks and gluons at low $x$, which can be accessed in the forward region. Here, $x$ is the fractional momentum carried by the parton. The first upgrade is a new forward electromagnetic calorimeter which will enable the measurement of neutral pions and photons at pseudorapidities from $1<\eta<3$. Measuring correlations between pions and direct photons at very forward angles will allow us to access the gluon distribution in deuteron-gold collisions at very low $x$, where saturation effects are expected to set in. In polarized proton collisions at $500 \mathrm{GeV}$, one goal of the RHIC spin program is the measurement of the sea quark polarizations via $\mathrm{W}$ production. Again, $\mathrm{W}$ bosons that are produced in the forward or backward direction probe the polarization of the sea-quarks. At PHENIX, these bosons can be detected in the leptonic decay channel in which a muon is detected in the muon arms which cover pseudorapidities from $1.2<|\eta|<2.2$. However, at present the trigger rejection rate of the experiment is not sufficient to trigger on this rare process which is characterized by a muon with a high $p_{T}$. Therefore, a trigger upgrade project is underway which consists of two parts: New front end electronics which will generate a fast trigger signal from the existing muon tracker wire chambers and dedicated resistive plate chambers (RPCs) that have sufficient time resolution to reject tracks that are not in time with tracks coming from the vertex, e.g. cosmic rays and muons travelling with the incoming beam.

\section{The Forward Calorimeter}

\subsection{Physics Goal}

The main physics goal of the proposed FOrward CALorimeter ( $\mathrm{FoCal})$ is to probe partons in the gold nucleus at an $x$ of about $10^{-3}-10^{-2}$. It is theorized that this regime is governed by the color glass condensate (CGC). The CGC [2] is a concept describing partons in cold nuclear matter that carry very little momentum so that the nuclear wavefunctions overlap, potentially giving rise to recombination and saturation effects. Since the CGC is opaque to color charges, one signature is the suppression of the away side peak in two jet events. This effect has already been seen by PHENIX and STAR in a limited kinematic range. The FoCal upgrade will dramatically increase PHENIX sensitivity in the forward region, where the effect is predicted to be large. This detector will be able to identify very forward $\pi^{0}$ 's and $\gamma$ 's, a capability which is unique at RHIC.

\subsection{Apparatus}

FoCal is a Tungsten-Silicon sampling calorimeter, approximately $16 \mathrm{~cm}\left(\approx 21 X_{0}\right)$ in depth. It is located $40 \mathrm{~cm}$ from the nominal interaction vertex and consists of $6.2 \times 6.2 \mathrm{~cm}$ supertowers, subdivided in $4 \times 4$ pads on the read-out silicon wafers. Longitudinally, it is split into three segments

of seven tungsten-silicon sandwiches, which are read out together, hence effectively summed. The 

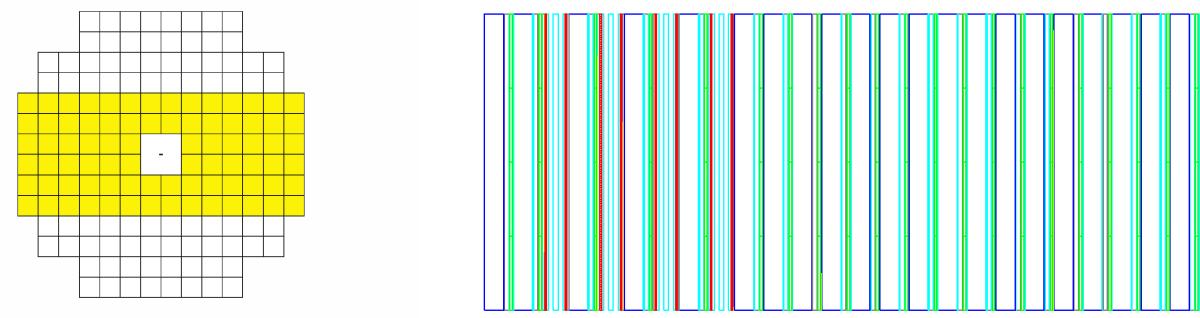

Figure 1: The FoCal acceptance. On the left - the beam view, on the right - the side view. Only the yellow area will be instrumented in the beginning. The innermost edge is at $\eta=1$, the outermost at $\eta=3$. Each square represents a $4 \times 4$ pad. The longitudinal view shows the tungsten (blue), silicon pads (green), and silicon strips (red).

first of these segments has eight additional silicon strip wafers for the identification of direct photons. These are pairs of $\mathrm{x}$ or $\mathrm{y}$ - oriented strips and there are $128 \mathrm{x} 1$ readouts per wafer. These strips are located at two, three, four, and five radiation length, where the shower is still in its infancy to optimize discrimination of direct $\gamma$ 's and $\pi^{0}$ 's which decay into two photons up to shower energies of about $50 \mathrm{GeV}$. The FoCal will cover a pseudorapidity range of $1<\eta<3$ but will be only partially instrumented in the beginning. Figure 1 shows the instrumented region.

The tracking capabilities in the first segment of the detector are novel and will enable, besides the global reconstruction of the shower energy and direction, good $\pi^{0} / \gamma$ discrimination. A Hough transformation will be used to reconstruct the slopes of the candidate tracks in the showers using seven points in space from the strip and pad layers. For pions, the distribution of the slopes will be bimodal, corresponding to the two decay photons. Showers initiated by a single direct photon will have only one peak. Isolated showers will have an energy resolution of $\Delta E / E \approx 23 \% / \sqrt{E}$ and an angular resolution of better than $50 \mu \mathrm{rad}$.

\section{The Muon Trigger}

An important part of the spin program at RHIC is the measurement of sea-quark polarizations in $\mathrm{W}$ production. The PHENIX detector can detect high momentum muons coming from $\mathrm{W}$ decays in the muon arms which cover a pseudorapidity of $1.2<\eta<2.2(2.4)$ on the south (north) side. They consist on each side of three stations of cathode strip chambers for tracking and outside of the magnetic field, a muon identifier station made up of 5 planes of Iarrocci streamer tubes with steel absorber planes in between. On the partonic level, the measured longitudinal, parity violating single spin asymmetry can be expressed by the longitudinal and unpolarized quark distribution functions:

$$
A_{L}^{W^{-}}=\frac{\sigma^{\rightarrow}-\sigma^{\leftarrow}}{\sigma^{\rightarrow}+\sigma^{\leftarrow}} \propto \frac{\Delta \bar{u}\left(x_{1}\right) d\left(x_{2}\right)-\Delta d\left(x_{1}\right) \bar{u}\left(x_{2}\right)}{d\left(x_{1}\right) \bar{u}\left(x_{2}\right)+\bar{u}\left(x_{1}\right) d\left(x_{2}\right)}
$$



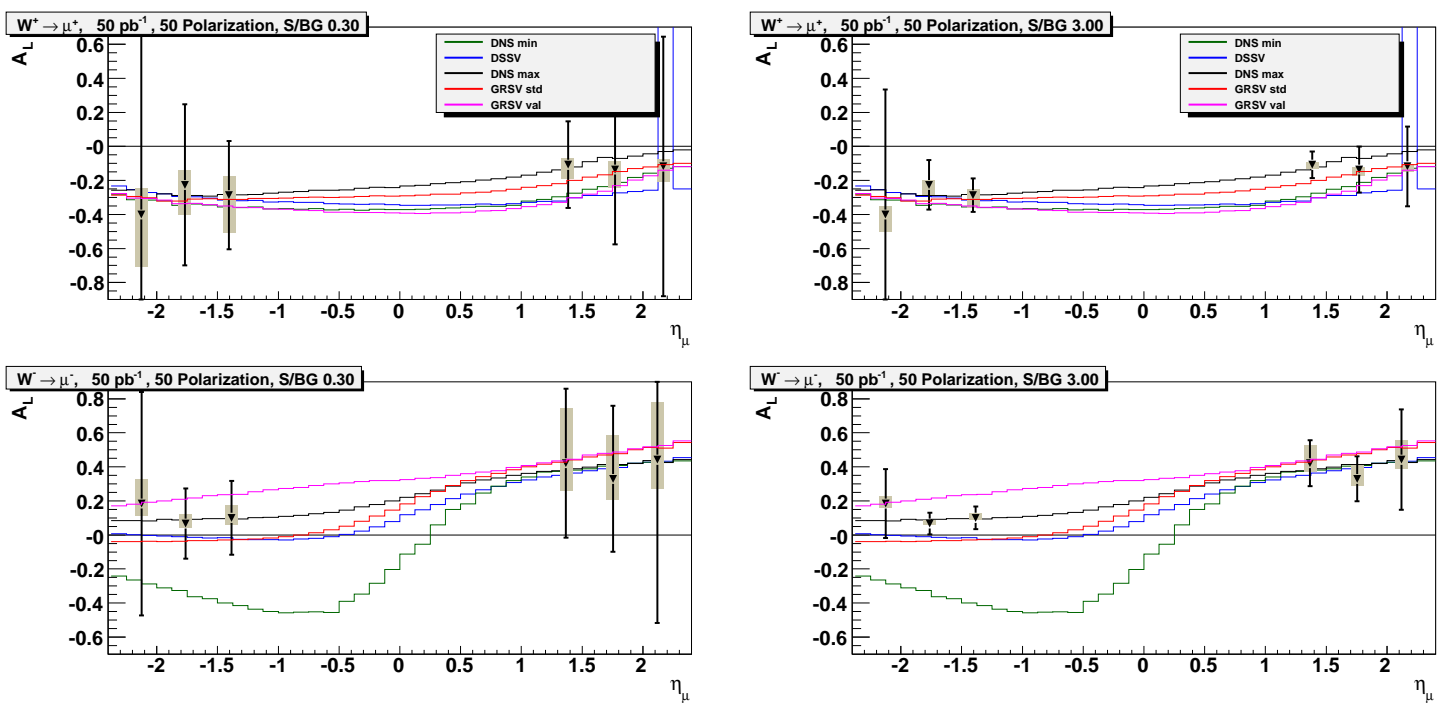

Figure 2: Predictions for the longitudinal spin asymmetry $A_{L}$ of the produced muon with respect to $\eta$ for signal to background ratios of 0.3 and 3.0. For comparison, predictions of current models are shown. Especially $\mu^{-}$asymmetries in the backward direction provide a high discriminating power, providing access to $\Delta \bar{u}$.

In the backward direction with respect to the parton carrying the momentum fraction $x_{1}$, contributions from $\Delta d \bar{u}$ are suppressed and $\Delta \bar{u}$ is probed more directly. The same is true for $W^{+}$ asymmetries in the forward direction. However, the decay muon smears this relation, especially for the $W^{+}$, where it is preferentially emitted opposite to the boson direction as described in [6].

Currently, PHENIX does not have the capability to effectively trigger on high momentum muons generated from W decays. Since this process is very rare, and only dominates over mouns from heavy quark decays above a $p_{T}$ of about $20 \mathrm{GeV}$, a new fast trigger system had to be implemented to achieve the required rejection factor of about 10000 by selecting high energy muons. The trigger system consists of two parts: New front end electronics for the existing MuTr stations, that use about $5 \%$ of the deposited charge to generate a fast trigger signal and new resistive plate chamber (RPC) detectors. Trigger signals from the two systems are then brought together in new level 1 trigger boards, generating a level 1 trigger signal if a straight track, indicating a high momentum muon, crossed the Muon Tracker and RPC stations.

While the new FEE will be able to generate a trigger signal fast enough to fix the beam crossing, which is every $106 \mathrm{~ns}$, the RPC with a timing resolution of better than $3 \mathrm{~ns}$ will enable the rejection of beam background and cosmic ray muons. At the same time, the detector fulfills the trigger requirements of an efficiency of more than $95 \%$ and a rate capability of more than $0.5 \mathrm{~Hz} / \mathrm{cm}^{2}$. Figure 3 shows the different parts of the trigger system schematically and a detailed description of the trigger system can be found in [4]. Resistive Plate Chambers are a well established technology, adopted by many experiments, including the major LHC experiments. The bakelite RPCs used by PHENIX follow the design of the CMS muon chambers. During RHIC run9 (2009/10), two prototypes with an adapted design were tested in $200 \mathrm{GeV}$ and $500 \mathrm{GeV}$ running. After the successful test, mass production of the detectors was started. Considering the experience of other 


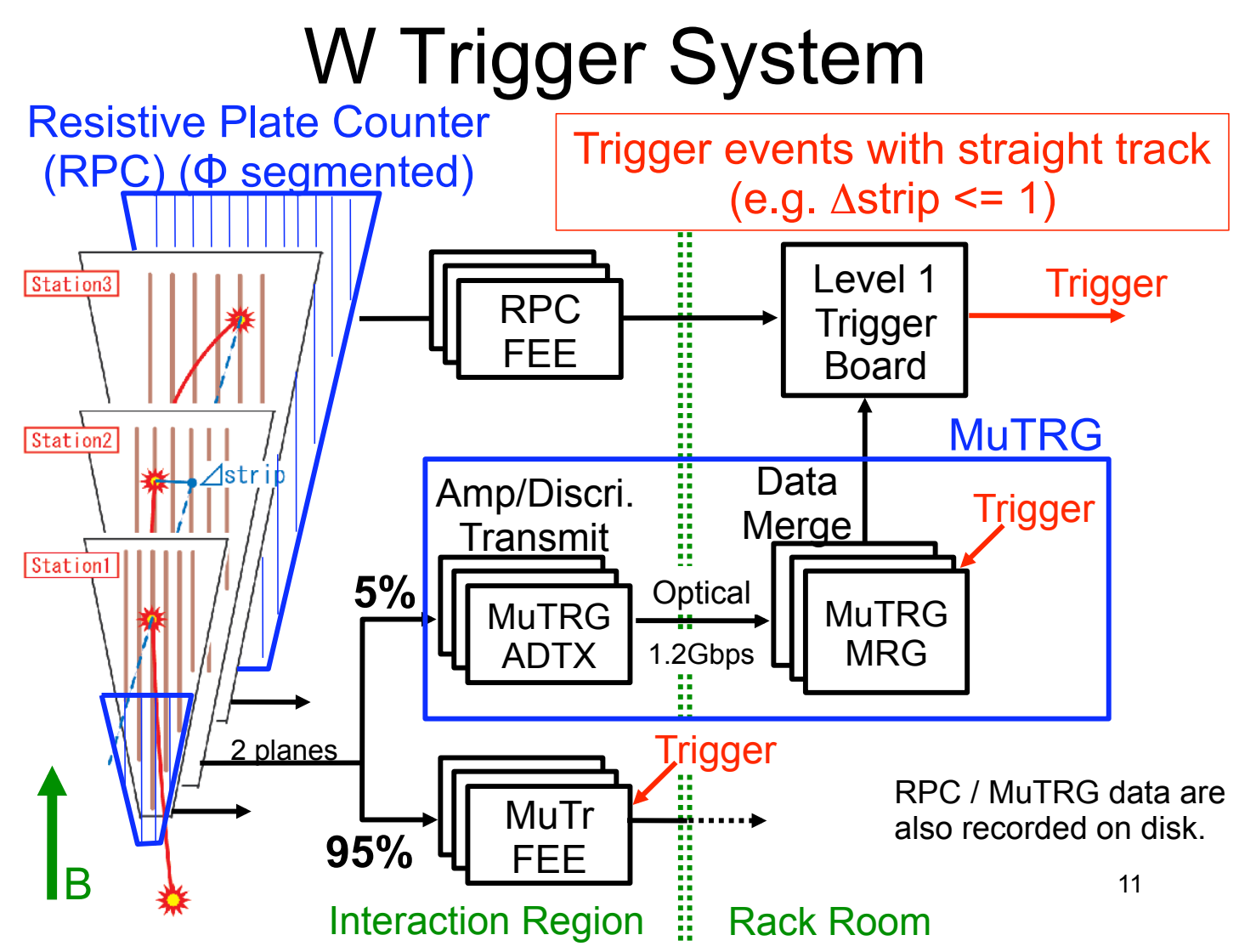

Figure 3: A schematic view of the muon trigger system. It consists of new amplifier/discriminator cards for the three existing muon tracker stations and two new RPC stations in each phenix muon arm.

experiments, a special emphasis has been put on quality control [5]. RPC station 3 on the north side was installed in November 2009 and the installation of station 3 on the south side is planned for this summer (2010) shutdown of RHIC. Additionally, $35 \mathrm{~cm}$ of steel absorber will be installed on both sides to minimize background due to punch-through hadrons.

Commissioning for the RPC 3 has been done using cosmic rays at the end of run 10 and will be continued with the upcoming $500 \mathrm{GeV}$ run 11. Parallel to the hardware development effort, extensive simulations have been performed, showing that the PHENIX setup will have very good discriminating power with respect to current models of the nucleon sea as shown in fig. 2.

\section{References}

[1] NIM A499 469-479 (2003)

[2] E. Iancu, A. Leonidov and L. McLerran, arXiv:hep-ph/0202270

[3] Conceptual Design Report for a Fast Muon Trigger (2007)

[4] Technical Design Report on Amplifier-Discriminator board and Data Transfer board for the MuTr FEE upgrade (2008)

[5] B. Hong, NIM A 602, 644-648 (2009)

[6] D. de Florian, W. Vogelsang, arXiv:1003.4533v2 